TRANSACTIONS OF THE

AMERICAN MATHEMATICAL SOCIETY

Volume 348, Number 5, May 1996

\title{
SIMULTANEOUS RATIONAL APPROXIMATION TO BINOMIAL FUNCTIONS
}

\author{
MICHAEL A. BENNETT
}

\begin{abstract}
We apply Padé approximation techniques to deduce lower bounds for simultaneous rational approximation to one or more algebraic numbers. In particular, we strengthen work of Osgood, $\mathrm{Fel}^{\prime} \mathrm{dman}$ and Rickert, proving, for example, that

$$
\max \left\{\left|\sqrt{2}-p_{1} / q\right|,\left|\sqrt{3}-p_{2} / q\right|\right\}>q^{-1.79155}
$$

for $q>q_{0}$ (where the latter is an effective constant). Some of the Diophantine consequences of such bounds will be discussed, specifically in the direction of solving simultaneous Pell's equations and norm form equations.
\end{abstract}

\section{INTRODUCTION}

In 1964, Baker [1, 2] utilized the method of Padé approximation to hypergeometric functions to obtain explicit improvements upon Liouville's theorem on rational approximation to algebraic numbers. By way of example, he showed that

$$
\left|\sqrt[3]{2}-\frac{p}{q}\right|>10^{-6} q^{-2.955}
$$

for all positive integers $p$ and $q$ and used such bounds to solve related Diophantine equations. Chudnovsky [6] subsequently refined Baker's results, primarily through a detailed analysis of the arithmetical properties of certain Padé approximants. Analogous to (0.1), he proved that

$$
\left|\sqrt[3]{2}-\frac{p}{q}\right|>q^{-2.42971}
$$

for all integers $p$ and $q$ with $q$ greater than some effectively computable constant $q_{0}$. By working out the implicit constants in (0.2), Easton [8] deduced

$$
\left|\sqrt[3]{2}-\frac{p}{q}\right|>6.6 \times 10^{-6} q^{-2.795}
$$

for positive integers $p$ and $q$ (as well as related bounds for other cubic irrationalities).

Similar results exist for simultaneous approximation to several algebraic numbers. In particular, Baker [3] derived bounds of the form

$$
\max _{1 \leq u \leq m}\left\{\left|\theta_{u}-\frac{p_{u}}{q}\right|\right\}>q^{-\lambda}
$$

Received by the editors June 30, 1994 and, in revised form, January 31, 1995.

1991 Mathematics Subject Classification. Primary 11J68, 11J82; Secondary 11D57.

Key words and phrases. Simultaneous approximation to algebraic numbers, irrationality and linear independence measures, Padé approximants, Pell-type equations, norm form equations.

Research supported by an NSERC Postdoctoral Fellowship. 
for certain algebraic numbers $\theta_{1}, \theta_{2}, \ldots, \theta_{m}$ with $1, \theta_{1}, \theta_{2}, \ldots, \theta_{m}$ linearly independent over the rationals, $\lambda=\lambda\left(\theta_{1}, \ldots, \theta_{m}\right)$ an explicit real number and $p_{1}, \ldots, p_{m}, q$ positive integers with $q$ greater than an effective $q_{0}\left(\lambda, \theta_{1}, \ldots, \theta_{m}\right)$. To be precise, he considered

$$
\left(\theta_{1}, \theta_{2}, \ldots, \theta_{m}\right)=\left(r^{\nu_{1}}, r^{\nu_{2}}, \ldots, r^{\nu_{m}}\right)
$$

with $r, \nu_{1}, \nu_{2}, \ldots, \nu_{m}$ rational, via approximation to the system of binomial functions

$$
1,(1+x)^{\nu_{1}}, \ldots,(1+x)^{\nu_{m}}
$$

Chudnovsky [6], generalizing his approach from the case of a single approximation, sharpened these inequalities. Along somewhat different lines, Osgood [13], Fel'dman [9] and Rickert [15] obtained results like (0.3) with

$$
\left(\theta_{1}, \theta_{2}, \ldots, \theta_{m}\right)=\left(r_{1}^{\nu}, r_{2}^{\nu}, \ldots, r_{m}^{\nu}\right)
$$

for $r_{1}, r_{2}, \ldots, r_{m}$ and $\nu$ rational. These also utilized Padé approximation, this time to the functions

$$
1,\left(1+a_{1} x\right)^{\nu}, \ldots,\left(1+a_{m} x\right)^{\nu}
$$

where $a_{1}, \ldots, a_{m}$ are distinct integers. Through use of an elegant contour integral representation for the desired Padé approximants, Rickert proved the inequality

$$
\max \left\{\left|\sqrt{2}-\frac{p_{1}}{q}\right|,\left|\sqrt{3}-\frac{p_{2}}{q}\right|\right\}>10^{-7} q^{-1.913}
$$

for $p_{1}, p_{2}$ and $q$ integral

In this paper, we will strengthen the work of Osgood, Fel'dman and Rickert on simultaneous approximation to algebraic numbers satisfying (0.5), in analogy to Chudnovsky's results for those with (0.4). This is primarily accomplished through careful estimation of both "analytic" and "arithmetic" asymptotics (in the same sense as Chudnovsky [6]) of Padé approximants to binomial functions. A particularly striking result along these lines (with rather different approximating forms) is due to Hata [11] who proved that

$$
\left|\pi-\frac{p}{q}\right| \geq q^{-8.0161}
$$

for sufficiently large positive integers $p$ and $q$.

In the special case $m=1$, we obtain Chudnovsky's Theorem 5.3 of [6] on approximation to a single algebraic number (see $\S 7$ ). For larger values of $m$, we can prove, for example, that

$$
\max \left\{\left|\sqrt{2}-\frac{p_{1}}{q}\right|,\left|\sqrt{3}-\frac{p_{2}}{q}\right|\right\}>q^{-1.79155}
$$

for $p_{1}$ and $p_{2}$ integral and $q \geq q_{0}$ effectively computable (compare to (0.6)). Similarly, we have

$$
\max \left\{\left|\sqrt{3}-\frac{p_{1}}{q}\right|,\left|\sqrt{5}-\frac{p_{2}}{q}\right|\right\}>q^{-1.82227}
$$

for $q \geq q_{1}$ effective. Optimally, one would like to derive (0.3) for any $\lambda>1+1 / m$. Theorems of Roth [16] $(m=1)$ and Schmidt [17] $(m>1)$ assert that such bounds exist for any independent algebraic $\theta_{1}, \ldots, \theta_{m}$, but are ineffective in that they do not permit the explicit calculation of $q_{0}$. For specific classes of algebraic numbers, however, we will be able to obtain effective bounds with $\lambda$ arbitrarily close to $1+1 / m$. These correspond to the situations described by previous authors where 
the rationals $r$ or $r_{1}, r_{2}, \ldots, r_{m}$ in (0.4) or (0.5), respectively, are suitably close to 1. We will also prove a theorem on linear forms, of the type

$$
\left|x_{0}+x_{1} \theta_{1}+\cdots+x_{m} \theta_{m}\right|>X^{-\lambda_{1}}
$$

for $x_{0}, \ldots, x_{m}$ integers, $\theta_{1}, \ldots, \theta_{m}$ as in (0.5) and $X=\max _{0 \leq i \leq m}\left|x_{i}\right|$ satisfying $X \geq$ $X_{0}\left(\lambda_{1}, \theta_{1}, \ldots, \theta_{m}\right)$. Standard transference arguments (see e.g. Cassels [5]) ensure that (0.3) implies (0.7) with exponent

$$
\lambda_{1}=\frac{m(\lambda-1)}{m(-\lambda+1)+\lambda}
$$

provided $\lambda<1+1 /(m-1)$. Our result, however, is somewhat stronger. These results have direct applications to Diophantine equations which we will address in $\S 8$ and $\S 9$. For example, they permit solution of the norm form equation

$$
N_{K / \mathbb{Q}}\left(x+y \sqrt[4]{M^{4}-1}+z \sqrt[4]{M^{4}+1}\right)=u
$$

(where $K=\mathbb{Q}\left(\sqrt[4]{M^{4}-1}, \sqrt[4]{M^{4}+1}\right), x, y$ and $z$ are integers and $u$ is constant) for $M \geq 6$.

\section{A PAIR OF THEOREMS ON RATIONAL APPROXIMATION}

Henceforth, we will suppose that $a_{0}, a_{1}, \ldots, a_{m}$ are distinct integers $(m \geq 1)$ with one of them equal to zero, satisfying

$$
a_{0}<a_{1}<\ldots<a_{m}
$$

Let us also assume that $N$ is a positive integer with

$$
N>\max _{0 \leq u \leq m}\left|a_{u}\right|
$$

and that $s$ and $n$ are integers with $1 \leq s<n$ and $(s, n)=1$. Define

$$
\begin{gathered}
c_{1}=\operatorname{lcm}\left\{\prod_{\substack{l=0 \\
l \neq v}}^{m}\left|a_{l}-a_{v}\right|: 0 \leq v \leq m\right\}, \\
c_{2}=\operatorname{lcm}\left\{\left|a_{l}-a_{v}\right|: 0 \leq v<l \leq m\right\}, \\
c_{3}=\prod_{p \mid n} p^{\max \left\{\operatorname{ord}_{p}\left(n / c_{2}\right)+\frac{1}{p-1}, 0,\right\}}
\end{gathered}
$$

and

\[ c_{4}=c_{1} \cdot c_{2} \cdot c_{3} \cdot \]
If, following Rickert [15], we set $A(z)=\prod_{u=0}^{m}\left(z-a_{u}\right)$, then the polynomial

$$
A(z)-(z+N) A^{\prime}(z)
$$

(where we write $A^{\prime}(z)$ for $d A(z) / d z$ ) is readily seen to have $m+1$ real zeros, one of them, say $z_{0}$, satisfying $z_{0}<-N$ and the remaining $m$, say $z_{1}, z_{2}, \ldots, z_{m}$, lying between successive values of the $a_{i}$ 's. Without loss of generality, we suppose that $a_{u-1}<z_{u}<a_{u}(1 \leq u \leq m)$ and define

$$
c_{5}=\left|A^{\prime}\left(z_{0}\right)\right|
$$




$$
c_{6}(v)= \begin{cases}\left|A^{\prime}\left(z_{1}\right)\right| & \text { if } v=0, \\ \min \left\{\left|A^{\prime}\left(z_{v}\right)\right|,\left|A^{\prime}\left(z_{v+1}\right)\right|\right\} & \text { if } 1 \leq v<m, \\ \left|A^{\prime}\left(z_{v}\right)\right| & \text { if } v=m,\end{cases}
$$

and

$$
c_{8}=\exp \left(-\gamma-\frac{1}{\phi(n)} \sum_{\substack{1 \leq r<n \\(r, n)=1}} \psi\left(\max \left\{\frac{n m-r}{n m}, \frac{r}{n}\right\}\right)\right)
$$

where $\gamma$ is Euler's constant, $\phi(n)$ is Euler's totient function and $\psi(z)=d \log \Gamma(z) / d z$ is the digamma function. We may conclude

Theorem 1.1. Let $a_{0}, a_{1}, \ldots, a_{m}$ be distinct integers with $a_{r}=0$ for some $r$ and $N>\max _{0 \leq u \leq m}\left|a_{u}\right|$ an integer. If, further, $s$ and $n$ are relatively prime with $1 \leq s<$ $n, \epsilon>0$ and $c_{7} \cdot c_{8}<c_{4}<c_{5} \cdot c_{8}$, then

$$
\max _{\substack{0 \leq u \leq m \\ a_{u} \neq 0}}\left\{\left|\left(1+\frac{a_{u}}{N}\right)^{s / n}-\frac{p_{u}}{q}\right|\right\}>q^{-\lambda-\epsilon}
$$

for all integers $p_{0}, \ldots, p_{m}$ and with $q \geq q_{0}\left(\epsilon, s, n, a_{0}, \ldots, a_{m}, N\right)$, where $\lambda=1+$ $\frac{\log \left(c_{4} / c_{7} \cdot c_{8}\right)}{\log \left(c_{5} \cdot c_{8} / c_{4}\right)}$ and $q_{0}$ is effectively computable.

As mentioned previously, in $\S 7$ we will show that, in the case $m=1$, the above theorem implies Chudnovsky's result [6, Theorem 5.3] (see also Heimonen, et al. [12]).

For linear forms, we will prove

Theorem 1.2. If $a_{0}, \ldots, a_{m}, N, s$ and $n$ are integers satisfying the hypotheses of the previous theorem, $x_{0}, \ldots, x_{m}$ integers, $X=\max _{0 \leq u \leq m}\left|x_{u}\right|, \epsilon>0$, and

$$
\prod_{v=1}^{m} c_{6}(v)<\left(c_{4} / c_{8}\right)^{m}<c_{5} \cdot \min _{1 \leq l \leq m} \prod_{\substack{v=1 \\ v \neq l}}^{m} c_{6}(v)
$$

then

$$
\left|\sum_{u=0}^{m} x_{u} \cdot\left(1+\frac{a_{u}}{N}\right)^{s / n}\right|>X^{-\lambda_{1}-\epsilon}
$$

for all $X \geq X_{0}\left(\epsilon, s, n, a_{0}, \ldots, a_{m}, N\right)$, where

$$
\lambda_{1}=\frac{m \log \left(c_{4} / c_{8}\right)-\sum_{1 \leq v \leq m} \log \left(c_{6}(v)\right)}{m \log \left(c_{8} / c_{4}\right)+\log \left(c_{5}\right)+\min _{1 \leq l \leq m} \sum_{\substack{1 \leq v \leq m \\ v \neq l}} \log \left(c_{6}(v)\right)}
$$

and $X_{0}$ is effectively computable.

Examples and applications of this result will be briefly described in $\S 8$ and $\S 9$. 


\section{The NATURE OF THE APPROXIMATING FORMS}

To construct our approximants to the system of binomial functions (0.3), we consider the contour integral

$$
I_{u}(x)=\frac{1}{2 \pi i} \int_{\gamma} \frac{(1+z x)^{k}(1+z x)^{\nu}}{\left(z-a_{u}\right)(A(z))^{k}} d z \quad(0 \leq u \leq m) .
$$

Here, $k$ is some fixed positive integer, $\nu$ a nonintegral positive rational, $\gamma$ a closed, counter-clockwise contour enclosing the poles of the integrand and $x$ a real satisfying

$$
|x|^{-1}>\max _{0 \leq u \leq m}\left|a_{u}\right| \text {. }
$$

By application of Cauchy's residue theorem, we can write

$$
I_{u}(x)=\sum_{v=0}^{m} P_{u v}(x)\left(1+a_{v} x\right)^{\nu} \quad(0 \leq u \leq m)
$$

where the $P_{u v}(x)$ are polynomials with rational coefficients and degree at most $k$ in $x$. Explicitly, from Rickert [15, Lemma 3.3], we have

$$
P_{u v}(x)=\sum\left(\begin{array}{c}
k+\nu \\
h_{v}
\end{array}\right)\left(1+a_{v} x\right)^{k-h_{v}} x^{h_{v}} \prod_{\substack{0 \leq l \leq m \\
l \neq v}}\left(\begin{array}{c}
-k_{u l} \\
h_{l}
\end{array}\right)\left(a_{v}-a_{l}\right)^{-k_{u l}-h_{l}}
$$

where $\sum$ denotes summation over all nonnegative integers $h_{0}, \ldots, h_{m}$ with sum $k_{u v}-1$, for $k_{a b}=k+\delta_{a b}$ and $\delta_{a b}$ the Kronecker delta. To guarantee the "independence" of the approximants, we require that $\operatorname{det}_{0 \leq u, v \leq m}\left(P_{u v}(x)\right)$ does not vanish for nonzero $x$, a consequence of Rickert's Lemma 3.4. To be precise, one may write

$$
\operatorname{det}_{0 \leq u, v \leq m}\left(P_{u v}(x)\right)=\left(\prod_{v=-1}^{m-1}\left(\begin{array}{c}
\nu-v k \\
k
\end{array}\right)\right) \cdot\left(\prod_{l=0}^{m} \prod_{\substack{s=0 \\
s \neq l}}^{m}\left(a_{s}-a_{l}\right)^{-k}\right) x^{(m+1) k} .
$$

In the sections that follow, we will find asymptotics for $\left|P_{u v}(1 / N)\right|$ and $\left|I_{u}(1 / N)\right|$ and then study the arithmetic properties of the coefficients of $P_{u v}(x)$.

\section{Contour integral estimates}

To begin, we note that the value $P_{u v}(x)\left(1+a_{v} x\right)^{\nu}(0 \leq u \leq m)$ is obtained from the integral (2.1), only with the contour $\gamma$ changed so as to enclose the integer $a_{v}$ and no other $a_{l}$ 's (for $l \neq v$ ). Setting $x=1 / N$, one sees that $(2.2)$ is satisfied and it follows that the integrand of (2.1) is analytic in a suitable deleted neighbourhood of $a_{v}$. Following Hata [11], we may apply the saddle-point method as described in Dieudonné $\left[7\right.$, chapter IX] to estimate the principal part of $P_{u v}\left(\frac{1}{N}\right)\left(1+a_{v} / N\right)^{\nu}$ for large values of $k$. Explicitly, we set

$$
F(z)=\log \left(1+\frac{z}{N}\right)-\log (|A(z)|)
$$

and

$$
G(z)=\left(1+\frac{z}{N}\right)^{\nu}\left(z-a_{u}\right)^{-1}
$$


so that

$$
P_{u v}\left(\frac{1}{N}\right)\left(1+a_{v} / N\right)^{\nu}=\int_{\gamma} G(z) e^{k F(z)} d z .
$$

The saddles of the surface $|F(z)|$ are given by the zeros of the derivative of $F(z)$ which, since $x=1 / N$, are the zeros of the polynomial (1.4) (say $z_{0}, z_{1}, \ldots, z_{m}$ as in $\S 1$ ). Since $G(z) e^{k F(z)}$ vanishes as $z$ tends to $-N$ or $\infty$ (avoiding the real branch cut from $-N$ to $-\infty$ ), the saddle-point method yields

Lemma 3.1. As $k \rightarrow \infty$, the principal part of is given by

$$
P_{u v}\left(\frac{1}{N}\right)\left(1+\frac{a_{v}}{N}\right)^{\nu} \sim \sum_{v \leq t \leq v+1} e^{k F\left(z_{t}\right)} G\left(z_{t}\right) \sqrt{\frac{-2 \pi}{k F^{\prime \prime}\left(z_{t}\right)}},
$$

where the summation is restricted to $t \in[1, m]$. In particular, since the roots of satisfy

$$
e^{F\left(z_{l}\right)}=\left|N A^{\prime}\left(z_{l}\right)\right|^{-1}(0 \leq l \leq m)
$$

we may conclude that

$$
\lim _{k \rightarrow \infty} \frac{1}{k} \log \left|P_{u v}\left(\frac{1}{N}\right)\right|=-\log \left(c_{6}(v) \cdot N\right) \leq-\log \left(c_{7} \cdot N\right)
$$

for all $0 \leq u, v \leq m$.

To find asymptotics for $\left|I_{u}(1 / N)\right|$ requires a more delicate analysis. Since the integrand of (2.1) has a branch point at $z=-N$, we cannot simply apply the saddle-point method for the saddle $z_{0}$ without justification (recall that $z_{0}<-N$ is real). If, however, we make the change of variables $1+\frac{z}{N} \rightarrow-w$, then we may write

$$
I_{u}\left(\frac{1}{N}\right)=\frac{(-1)^{m k} e^{\pi i \nu}}{2 \pi i N^{(m+1) k}} \int_{\gamma^{\prime}} \frac{w^{k} w^{\nu} d w}{\left(w+1+\frac{a_{u}}{N}\right)(B(w))^{k}}
$$

where $B(w)=\prod_{l=0}^{m}\left(w+1+\frac{a_{l}}{N}\right)$ and $\gamma^{\prime}=\gamma_{1}+\gamma_{2}+\gamma_{3}+\gamma_{4}$ is a contour containing the poles of the integrand of (3.2) while avoiding a branch cut along the nonnegative real axis (see Figure 1).

Since

$$
\left|\int_{\gamma_{l}} \frac{w^{k} w^{\nu} d w}{\left(w+1+\frac{a_{u}}{N}\right)(B(w))^{k}}\right| \leq \int_{0}^{2 \pi}\left|\frac{w^{k} w^{\nu}}{\left(w+1+\frac{a_{u}}{N}\right)(B(w))^{k}}\right| d \theta, \quad l=2 \text { or } 4
$$

(where $w=R e^{i \theta}$ or $r e^{i \theta}$ respectively), we have that the contribution to (3.2) associated with the arcs $\gamma_{2}$ and $\gamma_{4}$ becomes negligible as $r \rightarrow 0$ and $R \rightarrow \infty$. Therefore, from

$$
\frac{w^{k} w^{\nu}}{\left(w+1+\frac{a_{u}}{N}\right)(B(w))^{k}}=\left\{\begin{array}{l}
\frac{x^{k} x^{\nu}}{\left(x+1+\frac{a_{u}}{N}\right)(B(x))^{k}} \text { on } \gamma_{1}, \\
\frac{e^{2 \pi i \nu} x^{k} x^{\nu}}{\left(x+1+\frac{a_{u}}{N}\right)(B(x))^{k}} \text { on } \gamma_{3},
\end{array}\right.
$$




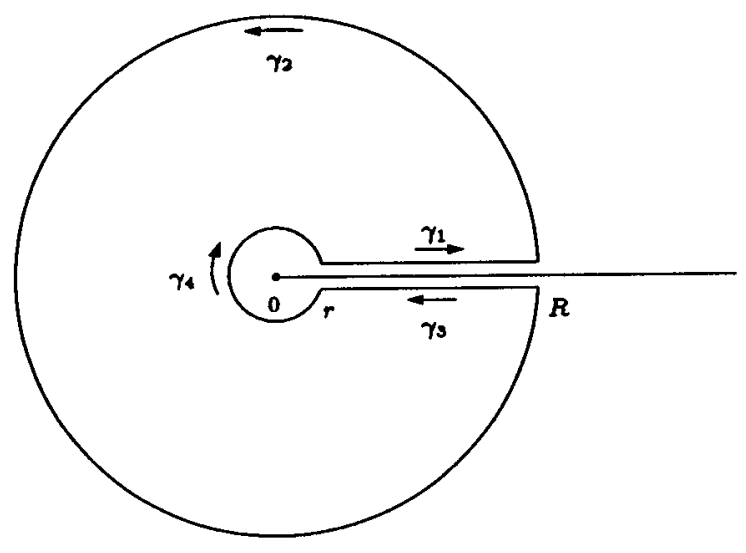

Figure 1

we may conclude, letting $r \rightarrow 0$ and $R \rightarrow \infty$, that

$$
I_{u}\left(\frac{1}{N}\right)=\frac{(-1)^{m k} e^{\pi i \nu}\left(1-e^{2 \pi i \nu}\right)}{2 \pi i N^{(m+1) k}} \int_{0}^{\infty} \frac{x^{k} x^{\nu} d x}{\left(x+1+\frac{a_{u}}{N}\right)(B(x))^{k}} .
$$

This is readily evaluated for large $k$ via Laplace's method (see e.g. Dieudonné [7, Chapter IV, §2]). Since the function $x / B(x)$ has only one critical point on the positive real axis, say $x_{0}$, and vanishes as $x \rightarrow 0^{+}$or $x \rightarrow \infty$, we conclude that

$$
\lim _{k \rightarrow \infty} \frac{1}{k} \log \left|I_{u}\left(\frac{1}{N}\right)\right|=\log \left|\frac{x_{0}}{N^{m+1} B\left(x_{0}\right)}\right| .
$$

Changing variables back to our original $z$, we find that

$$
\left|\frac{x_{0}}{N^{m+1} B\left(x_{0}\right)}\right|=\left|\frac{1+z_{0} / N}{A\left(z_{0}\right)}\right|=\left|\frac{1}{N A^{\prime}\left(z_{0}\right)}\right|
$$

for $z_{0}$ as in $\S 1$. Combining this with (3.3) yields

Lemma 3.2. $\lim _{k \rightarrow \infty} \frac{1}{k} \log \left|I_{u}\left(\frac{1}{N}\right)\right|=-\log \left(c_{5} \cdot N\right)(0 \leq u \leq m)$.

\section{Coefficients of the approximating polynomials}

We wish to determine certain arithmetic properties of the coefficients of the polynomials $P_{u v}(x)$ defined in $\S 2$. Let us write

$$
P_{v}=\prod_{\substack{0 \leq l \leq m \\
l \neq \neq}}\left(\begin{array}{c}
k+h_{l}-1 \\
h_{l}
\end{array}\right)\left(a_{l}-a_{v}\right)^{-k-h_{l}} .
$$

From (2.4), if $u=v$, then

$$
P_{u v}(x)=(-1)^{m k} \sum\left(\begin{array}{c}
k+\nu \\
h_{v}
\end{array}\right)\left(1+a_{v} x\right)^{k-h_{v}} x^{h_{v}} P_{v}
$$


where $\sum$ implies the sum over nonnegative $h_{0}, \ldots, h_{m}$ satisfying $\sum_{l=0}^{m} h_{l}=k$. If, however, $u \neq v$, we have

$$
P_{u v}(x)=(-1)^{m k} \sum\left(\begin{array}{c}
k+\nu \\
h_{v}
\end{array}\right)\left(1+a_{v} x\right)^{k-h_{v}} x^{h_{v}}\left(\frac{k+h_{u}}{k\left(a_{u}-a_{v}\right)}\right) P_{v}
$$

where in this latter case, the summation is over nonnegative $h_{0}, \ldots, h_{m}$ with $\sum_{l=0}^{m} h_{l}=k-1$. From here on we will fix $\nu=s / n$. The following elementary lemma concerning primes dividing binomial coefficients will be the chief tool in determining our "arithmetic" asymptotics. It enables us to identify certain classes of prime numbers which are guaranteed to divide the numerators of the coefficients of the polynomials $P_{u v}(x)$ defined in (2.4). Similar results have been utilized by Chudnovsky [6], Hata [11] and Heimonen, et al. [12], amongst others, in the pursuit of irrationality and linear independence measures. Suppressing dependence on $m, n, s$ and $k$, let

$$
\begin{aligned}
S(r)= & \{p \text { prime }: p>\sqrt{n k+s},(p, n k)=1(\text { and, if } m=1, \\
& (p, n k-s-n)=1) \text { and }\left\{\frac{k-1}{p}\right\}>\max \left(\frac{n m-r}{n m}, \frac{r}{n}\right) \\
& \text { for } r \text { with } 1 \leq r<n \text { and } p r \equiv s(\bmod n)\}
\end{aligned}
$$

where we adopt the notation $\{x\}=x-[x]$ for the fractional part of a real number $x$.

Lemma 4.1. If $p \in S(r)$, then

$$
\operatorname{ord}_{p}\left(\left(\begin{array}{c}
k+s / n \\
h_{0}
\end{array}\right)\left(\begin{array}{c}
k+h_{1}-1 \\
h_{1}
\end{array}\right) \cdots\left(\begin{array}{c}
k+h_{m}-1 \\
h_{m}
\end{array}\right)\right) \geq 1
$$

for all nonnegative integers $h_{0}, \ldots, h_{m}$ with $\sum_{l=0}^{m} h_{l}=k$ or $k-1$.

Proof. Suppose that $p \in S(r)$ does not divide the product

$$
\left(\begin{array}{c}
k+h_{1}-1 \\
h_{1}
\end{array}\right) \cdots\left(\begin{array}{c}
k+h_{m}-1 \\
h_{m}
\end{array}\right) .
$$

Then it follows that

$$
\left\{\frac{h_{l}}{p}\right\}<1-\left\{\frac{k-1}{p}\right\} \quad(1 \leq l \leq m)
$$

and thus, since $p \nmid k$, that

$$
\left\{\frac{h_{l}}{p}\right\} \leq 1-\left\{\frac{k}{p}\right\} \quad(1 \leq l \leq m)
$$

From (4.3), we may therefore write

$$
\sum_{l=1}^{m}\left\{\frac{h_{l}}{p}\right\} \leq m\left(1-\left\{\frac{k}{p}\right\}\right)<m\left(\frac{r}{n m}-\frac{1}{p}\right)=\frac{r}{n}-\frac{m}{p} .
$$


Hence $\sum_{l=0}^{m} h_{l}=k$ or $k-1$ in conjunction with

$$
\left\{\frac{k-1}{p}\right\}>\frac{r}{n}>\sum_{l=1}^{m}\left\{\frac{h_{l}}{p}\right\}
$$

yields

$$
\left\{\frac{k-h_{0}}{p}\right\} \leq \sum_{l=1}^{m}\left\{\frac{h_{l}}{p}\right\}+\frac{1}{p}
$$

We wish to show that $\operatorname{ord}_{p}\left(\begin{array}{c}k+s / n \\ h_{0}\end{array}\right) \geq 1$. Since $p \nmid n$, we have

$$
\operatorname{ord}_{p}\left(\begin{array}{c}
k+s / n \\
h_{0}
\end{array}\right)=\operatorname{ord}_{p}\left(\frac{(n k+s)(n(k-1)+s) \cdots\left(n\left(k-h_{0}\right)+n+s\right)}{h_{0} !}\right)
$$

and so by a result of Chudnovsky [6, Lemma 4.5] (recalling that $p>\sqrt{n k+s}$ )

$$
\operatorname{ord}_{p}\left(\begin{array}{c}
k+s / n \\
h_{0}
\end{array}\right)=\left\{\frac{k-\theta-h_{0}}{p}\right\}+\left\{\frac{h_{0}}{p}\right\}-\left\{\frac{k-\theta}{p}\right\}
$$

where $\theta=\frac{p r-s}{n}$. We thus have that $\operatorname{ord}_{p}\left(\begin{array}{c}k+s / n \\ h_{0}\end{array}\right) \geq 1$ exactly when $\left\{\frac{h_{0}}{p}\right\}>$ $\left\{\frac{k-\theta}{p}\right\}$ or, equivalently, when

$$
\left\{\frac{k-h_{0}}{p}\right\}<\frac{r}{n}-\frac{s}{p n} .
$$

Now, combining (4.5) and (4.6) gives

$$
\left\{\frac{k-h_{0}}{p}\right\} \leq \frac{r}{n}-\frac{(m-1)}{p}
$$

which for $m \geq 2$ implies $\left\{\frac{k-h_{0}}{p}\right\} \leq \frac{r}{n}-\frac{1}{p}<\frac{r}{n}-\frac{s}{p n}$, as desired (since $1 \leq s<n$ ). It remains to consider the case $m=1$. If we have strict inequality in (4.4), then

$$
\left\{\frac{h_{1}}{p}\right\} \leq 1-\left\{\frac{k}{p}\right\}-\frac{1}{p}<\frac{r}{n}-\frac{2}{p}
$$

whence

$$
\left\{\frac{k-h_{0}}{p}\right\} \leq\left\{\frac{h_{1}}{p}\right\}+\frac{1}{p}<\frac{r}{n}-\frac{1}{p}
$$

which again implies (4.8). Similarly, we attain the inequality (4.8) if $h_{0}+h_{1}=k$. If $\left\{\frac{h_{0}}{p}\right\}<\left\{\frac{k-\theta}{p}\right\}$, then

$$
\left\{\frac{k-h_{0}}{p}\right\} \geq \frac{r}{n}-\frac{s}{p n}+\frac{1}{p}>\frac{r}{n}
$$

contradicting (4.9). To complete the proof of the lemma, we have only to show that the simultaneous equalities

$$
\left\{\frac{h_{1}}{p}\right\}=1-\left\{\frac{k}{p}\right\}
$$




$$
\left\{\frac{h_{0}}{p}\right\}=\left\{\frac{k-\theta}{p}\right\}
$$

and

$$
h_{0}+h_{1}=k-1
$$

produce a contradiction. Well, the first of these implies that $p \mid k+h_{1}$, while the second yields $p \mid n\left(k-h_{0}\right)+s$. But these together with $h_{0}+h_{1}=k-1$ imply that $p$ divides $n k-s-n$, contradicting our initial assumptions.

Now if by $\pi(x, n, s)$ we denote the number of primes $p \leq x$ in the arithmetic progression $b n+s$, then, analogous to the standard prime number theorem, we have

$$
\pi(x, n, s)=\frac{x}{\phi(n) \log x}\left(1+O\left(\frac{1}{\log x}\right)\right) .
$$

It follows that if $\beta>\alpha>0$, then

$$
\lim _{k \rightarrow \infty} \frac{1}{k} \log \prod p=\frac{\beta-\alpha}{\phi(n)}
$$

where the product is over all primes $p$ in the interval $\alpha k<p<\beta k$ and satisfying $p \equiv a(\bmod n)$. The inequality $\left\{\frac{k-1}{p}\right\}>\max \left(\frac{n m-r}{n m}, \frac{r}{n}\right)$ defines a collection of open intervals for primes $p$ in $S(r)$, of the form

$$
\left(\frac{k}{l+1}, \min \left(\frac{n m k}{(l+1) n m-r}, \frac{n k}{l n+r}\right)+O(\sqrt{k})\right), \quad l=0,1,2, \ldots,
$$

where the shape of the error term follows from the assumption that $p>\sqrt{n k+s}$. From(4.10), we may therefore write

$$
\lim _{k \rightarrow \infty} \frac{1}{k} \log \prod_{p \in S(r)} p=\frac{1}{\phi(n)} \sum_{l=0}^{\infty}\left(\min \left(\frac{n m}{(l+1) n m-r}, \frac{n}{l n+r}\right)-\frac{1}{l+1}\right) .
$$

Since, from Bateman and Erdélyi [4], the function $\psi(z)=\frac{d \log \Gamma(z)}{d z}$ satisfies

$$
\psi(\beta)-\psi(\alpha)=\sum_{l=0}^{\infty}\left(\frac{1}{l+\alpha}-\frac{1}{l+\beta}\right),
$$

we may conclude that

$$
\lim _{k \rightarrow \infty} \frac{1}{k} \log \prod_{p \in S(r)} p=\frac{1}{\phi(n)}\left(\psi(1)-\psi\left(\max \left\{\frac{n m-r}{n m}, \frac{r}{n}\right\}\right)\right)
$$

whence, recalling the equality $\psi(1)=-\gamma$,

$$
\lim _{k \rightarrow \infty} \frac{1}{k} \log \prod_{\substack{1 \leq r<n \\(r, n)=1}} \prod_{p \in S(r)} p=-\gamma-\frac{1}{\phi(n)} \sum_{\substack{1 \leq r<n \\(r, n)=1}} \psi\left(\max \left\{\frac{n m-r}{n m}, \frac{r}{n}\right\}\right) .
$$

We thus have 
Lemma 4.2. For each $k$, there is a rational number $C_{k}$ such that

$$
C_{k} P_{u v}\left(\frac{1}{N}\right) \in \mathbb{Z} \text { for all } 0 \leq u, v \leq m
$$

and

$$
\lim \sup _{k \rightarrow \infty} \frac{1}{k} \log \left|C_{k}\right| \leq \log \left(c_{4} N / c_{8}\right)
$$

for $c_{4}$ and $c_{8}$ as in $\S 1$.

Proof. If we define $\mu_{n, r}=\prod_{p \mid n} p^{[r /(p-1)]}$, then by Chudnovsky [6, Lemma 4.1], we have

$$
\mu_{n, r} \cdot n^{r}\left(\begin{array}{c}
k+s / n \\
r
\end{array}\right) \in \mathbb{Z}
$$

for $k \geq r \geq 0$. Let

$$
\alpha=\max _{0 \leq l<v \leq m} \operatorname{ord}_{p}\left(a_{l}-a_{v}\right)
$$

and

$$
\beta_{v}\left(h_{0}, \ldots, h_{m}\right)=\mu_{n, h_{v}} \cdot n^{h_{v}} \prod_{\substack{0 \leq l \leq m \\ l \neq v}}\left(a_{l}-a_{v}\right)^{h_{l}}
$$

where $\sum_{0 \leq l \leq m} h_{l}=k$ or $k-1$.

If $p$ is prime and $p$ does not divide $n$, then

$$
\begin{aligned}
& \operatorname{ord}_{p}\left(\beta_{v}\left(h_{0}, \ldots, h_{m}\right)\right)=\operatorname{ord}_{p} \prod_{\substack{0 \leq l \leq m \\
l \neq j \\
l \neq v}}\left(a_{l}-a_{v}\right)^{h_{l}} \\
& =\sum_{\substack{0 \leq l \leq m \\
l \neq v}} h_{l} \operatorname{ord}_{p}\left(a_{l}-a_{v}\right) \leq \sum_{0 \leq l \leq m} \alpha h_{l} \leq \alpha k .
\end{aligned}
$$

On the other hand, if $p \mid n$, then

$$
\begin{aligned}
& \operatorname{ord}_{p}\left(\beta_{v}\left(h_{0}, \ldots, h_{m}\right)\right) \leq\left[\frac{h_{v}}{p-1}\right]+h_{v} \operatorname{ord}_{p} n+\sum_{\substack{0 \leq l \leq m \\
l \neq v}} h_{l} \operatorname{ord}_{p}\left(a_{l}-a_{v}\right) \\
& \leq h_{v}\left(\operatorname{ord}_{p} n+\frac{1}{p-1}\right)+\sum_{\substack{0 \leq l \leq m \\
l \neq v}} \alpha h_{l} \\
& \leq \max \left(\operatorname{ord}_{p} n+\frac{1}{p-1}, \alpha\right) \sum_{\substack{l \neq l \leq m \\
0 \leq l \leq m}} h_{l} \\
& \leq \max \left(\operatorname{ord}_{p} n+\frac{1}{p-1}, \alpha\right) k .
\end{aligned}
$$

Since $h_{l} \leq k$ for $0 \leq l \leq m,(1.1)$ and (1.2) yield that

$$
\beta_{v}\left(h_{0}, \ldots, h_{m}\right)^{-1} \cdot\left(c_{2} \cdot c_{3}\right)^{k}
$$

is an integer for all $0 \leq v \leq m$ and nonnegative integers $h_{0}, \ldots, h_{m}$ with sum $k-1$, or $k$. 
From (4.1), (4.2), (4.12), (4.13) and Lemma 4.1, we therefore have that

$$
\left(c_{4} \cdot N\right)^{k} \cdot c_{2} \cdot k\left(\prod_{\substack{1 \leq r \leq n \\(r, n)=1}} \prod_{p \in S(r)} p\right)^{-1} P_{u v}\left(\frac{1}{N}\right)
$$

is also an integer for $0 \leq u, v \leq m$ (using the fact that $P_{u v}(x)$ has degree at most $k)$. The lemma then follows from equality (4.11).

\section{Proof of Theorem 1.1}

To apply the results of the previous sections, we first state a lemma that connects our approximating polynomials with bounds of the form $(0.1)$. Let $\theta_{0}, \theta_{1}, \ldots, \theta_{m}$ be distinct real numbers with $\theta_{r}=1$ for some $r$. Then

Lemma 5.1. Suppose there are positive real numbers $P$ and $Q$ such that, for $\epsilon>0$ and each positive integer $k$ greater than an effective constant $k_{0}$, we can find integers $P_{u v k}(0 \leq u, v \leq m)$ with nonzero determinant,

$$
\frac{1}{k} \log \left|P_{u v k}\right| \leq P+\epsilon(0 \leq u, v \leq m)
$$

and

$$
\frac{1}{k} \log \left|\sum_{v=0}^{m} P_{u v k} \theta_{v}\right| \leq-Q+\epsilon(0 \leq u \leq m) .
$$

Then (0.1) holds for any $\lambda>1+\frac{P}{Q}$ and $q \geq q_{0}\left(\theta_{0}, \ldots, \theta_{m}, \lambda\right)$ effectively computable.

Proof. This follows directly from Lemma 2.1 in [14].

To complete the proof of Theorem 1.1, we write

$$
\theta_{l}=\left(1+\frac{a_{l}}{N}\right)^{s / n} \quad(0 \leq l \leq m)
$$

and

$$
P_{u v k}=C_{k} P_{u v}\left(\frac{1}{N}\right) \quad(0 \leq u, v \leq m)
$$

where $C_{k}$ is as in Lemma 4.2. Then from Lemmas 3.1 and 4.2, we may take

$$
P=\log \left(\frac{c_{4}}{c_{7} \cdot c_{8}}\right)
$$

and

$$
Q=\log \left(\frac{c_{5} \cdot c_{8}}{c_{4}}\right)
$$

in Lemma 5.1. If $c_{7} \cdot c_{8}<c_{4}<c_{5} \cdot c_{8}$, then both of these quantities are positive and, recalling that $\operatorname{det}_{0 \leq u, v \leq m}\left(P_{u v}(1 / N)\right)$ is nonzero, we can utilize this last lemma to obtain the desired result. Since we can make Lemma 5.1 effective, the same is true for Theorem 1.1. 


\section{Proof of Theorem 1.2}

Let $x_{0}, \ldots, x_{m}, a_{0}, \ldots, a_{m}, s, n, N$ and $X$ be as in $\S 1, C_{k}$ and $P_{u v k}$ as in $\S 4$ and $\S 5$ and write

$$
L=\sum_{u=0}^{m} x_{u} \cdot\left(1+\frac{a_{u}}{N}\right)^{s / n}
$$

Then the fact that $\operatorname{det}_{0 \leq u, v \leq m}\left(P_{u v}(1 / N)\right) \neq 0$ implies that we can find $m$ of the $C_{k} I_{u}(1 / N)(0 \leq u \leq m)$, say $C_{k} I_{1}(1 / N), \ldots, C_{k} I_{m}(1 / N)$ with

$$
L, C_{k} I_{1}\left(\frac{1}{N}\right), \ldots, C_{k} I_{m}\left(\frac{1}{N}\right)
$$

independent forms in the numbers $\left(1+a_{0} / N\right)^{s / n}, \ldots,\left(1+a_{m} / N\right)^{s / n}$. Following Fel'dman [9], we consider the determinant

$$
\Delta_{k}=\left|\begin{array}{llll}
x_{0} & x_{1} & \ldots & x_{m} \\
P_{10 k} & P_{11 k} & \ldots & P_{1 m k} \\
\cdot & \cdot & & \cdot \\
\cdot & \cdot & & \cdot \\
\cdot & \cdot & & \cdot \\
\cdot & \cdot & & \cdot \\
P_{m 0 k} & P_{m 1 k} & \ldots & P_{m m k}
\end{array}\right|=L \cdot \Delta_{k, 0}+\sum_{l=1}^{m} C_{k} I_{l}\left(\frac{1}{N}\right) \Delta_{k, l}
$$

where $\Delta_{k, l}(0 \leq l \leq m)$ are the cofactors of the elements of the first column of $\Delta_{k}$. Since the $P_{u v k}$ 's and $x_{v}$ 's are integers, the independence of the forms in (6.1) ensures that $\left|\Delta_{k}\right| \geq 1$ and thus (6.2) implies that

$$
|L| \cdot\left|\Delta_{k, 0}\right|+\sum_{l=1}^{m}\left|C_{k} I_{l}(1 / N)\right| \cdot\left|\Delta_{k, l}\right| \geq 1 .
$$

Define, for $\sigma \in S_{m}$ (i.e. $\sigma$ a permutation on $\{1,2, \ldots, m\}$ ) and $v=1,2, \ldots, m$,

$$
P(\sigma)=P_{\sigma(1) 1 k} P_{\sigma(2) 2 k} \ldots P_{\sigma(m) m k}
$$

and

$$
P_{v}(\sigma)=P_{\sigma(1) 1 k} \ldots P_{\sigma(v-1)(v-1) k} P_{\sigma(v+1)(v+1) k} \ldots P_{\sigma(m) m k}
$$

Since

we have that

$$
\Delta_{k, 0}=\left|\begin{array}{lll}
P_{11 k} & \ldots & P_{1 m k} \\
\cdot & & \cdot \\
\cdot & & \cdot \\
\cdot & & \cdot \\
\cdot & & \cdot \\
P_{m 1 k} & \ldots & P_{m m k}
\end{array}\right|
$$

$$
\left|\Delta_{k, 0}\right| \leq m ! \cdot \max \left\{|P(\sigma)|: \sigma \in S_{m}\right\}
$$

and thus Lemmas 3.1 and 4.2 yield

$$
\lim \sup _{k \rightarrow \infty} \frac{1}{k} \log \left|\Delta_{k, 0}\right| \leq m \log \left(\frac{c_{4}}{c_{8}}\right)-\sum_{v=1}^{m} \log \left(c_{6}(v)\right) .
$$


Similarly, if $1 \leq l \leq m$, then $\Delta_{k, l}$ satisfies

$$
\left|\Delta_{k, l}\right| \leq(m-1) ! \cdot \sum_{v=1}^{m}\left|x_{v}\right| \cdot \max \left\{\left|P_{v}(\sigma)\right|: \sigma \in S_{m}\right\}
$$

and Lemmas 3.2 and 4.2 imply (for $1 \leq v, l \leq m$ ) that

$$
\begin{gathered}
\lim \sup _{k \rightarrow \infty} \frac{1}{k} \log \left(C_{k} I_{l}\left(\frac{1}{N}\right) \max \left\{\left|P_{v}(\sigma)\right|: \sigma \in S_{m}\right\}\right) \\
\leq m \log \left(\frac{c_{4}}{c_{8}}\right)-\log \left(c_{5}\right)-\min _{1 \leq l \leq m} \sum_{\substack{v=1 \\
v \neq l}}^{m} \log \left(c_{6}(v)\right) .
\end{gathered}
$$

Let us denote the right-hand side of (6.4) by $D_{1}$ and the right-hand side of (6.5) by $D_{2}$. Given $\epsilon_{1}>0$, from (6.4) we can find a $k_{0}=k_{0}\left(\epsilon_{1}\right)$ for which

$$
\left|\Delta_{k, 0}\right| \leq \frac{1}{2} \cdot e^{\left(D_{2}+\epsilon_{1}\right) k}
$$

for $k \geq k_{0}$. Inequality (6.5) implies that for $X \geq X_{0}\left(\epsilon_{1}\right)$, we may find a $k=k(X)$ with both

$$
m ! \cdot \sum_{l=1}^{m}\left|C_{k} I_{l}\left(\frac{1}{N}\right)\right| \cdot \max \left\{\left|P_{v}(\sigma)\right|: \sigma \in S_{m}, 1 \leq v \leq m\right\} \leq \frac{1}{2 X}
$$

and

$$
X \leq e^{-\left(D_{1}-\epsilon_{1}\right) k} .
$$

Now (6.3) and (6.7) yield $|L| \cdot\left|\Delta_{k, 0}\right| \geq 1 / 2$ whence

$$
|L| \geq \frac{1}{2\left|\Delta_{k, 0}\right|} \geq e^{-\left(D_{2}+\epsilon_{1}\right) k}
$$

via (6.6). Applying (6.8), then, gives

$$
|L| \geq X^{\frac{D_{2}+\epsilon_{1}}{D_{1}-\epsilon_{1}}} \geq X^{D_{2} / D_{1}+\epsilon}
$$

for suitably small $\epsilon_{1}$ relative to $\epsilon$ and $X \geq X_{0}(\epsilon)$. This completes the proof of Theorem 1.2.

\section{The CASE Of a Single Binomial Function}

In the following analysis, we specialize Theorem 1.1 by fixing $m=1$, and writing $a_{1}=a$ (the ordering of the $a_{u}$ 's, as given in $\S 1$, is unimportant here). Further, take $n \geq 3$ and define

$$
c_{9}=\prod_{p \mid n} p^{\min \left\{\operatorname{ord}_{p} a, \operatorname{ord}_{p} n+\frac{1}{p-1}\right\}}
$$

and

$$
c_{10}=\exp \left(\frac{1}{\phi(n)} \sum_{\substack{1 \leq r<n / 2 \\(r, n)=1}}\left(\psi\left(\frac{n-r}{n}\right)-\psi\left(\frac{r}{n}\right)\right)\right) .
$$

We then obtain the following result of Chudnovsky [6, Theorem 5.3] as a corollary to Theorem 1.1: 
Theorem 7.1. If $a, N, s$ and $n$ are integers with $N>|a|, 1 \leq s<n,(s, n)=1, \epsilon>$ 0 and $(\sqrt{N+a}-\sqrt{N})^{2} \cdot c_{10}<c_{9}$, then

$$
\left|\left(1+\frac{a}{N}\right)^{s / n}-\frac{p}{q}\right|>q^{-\lambda-\epsilon}
$$

for $p$ and $q$ integers with $q \geq q_{0}(\epsilon, a, N, s, n)$ and

$$
\lambda=1-\frac{\log \left\{(\sqrt{N+a}+\sqrt{N})^{2} \cdot c_{10} / c_{9}\right\}}{\log \left\{(\sqrt{N+a}-\sqrt{N})^{2} \cdot c_{10} / c_{9}\right\}} .
$$

Proof. From (1.1) and (1.2), we have that $c_{1}=c_{2}=a$. Since $A(z)=z(z-a)$, the zeros of polynomial (1.4) are given by $z_{0}=-N-\sqrt{N^{2}+a N}$ and $z_{1}=-N+$ $\sqrt{N^{2}+a N}$. It follows that

$$
c_{5}=2 N+a+2 \sqrt{N^{2}+a N}=(\sqrt{N+a}+\sqrt{N})^{2}
$$

and

$$
c_{7}=\left|2 N+a-2 \sqrt{N^{2}+a N}\right|=(\sqrt{N+a}-\sqrt{N})^{2}
$$

and so we may apply Theorem 1.1 to deduce a bound of the form (7.1) with

$$
\begin{aligned}
\lambda & =1+\frac{\log \left(c_{4} / c_{7} \cdot c_{8}\right)}{\log \left(c_{5} \cdot c_{8} / c_{4}\right)} \\
& =1+\frac{\log \left(a^{2} \cdot c_{3} /(\sqrt{N+a}-\sqrt{N})^{2} \cdot c_{8}\right)}{\log \left((\sqrt{N+a}+\sqrt{N})^{2} \cdot c_{8} / a^{2} \cdot c_{3}\right)} \\
& =1-\frac{\log \left((\sqrt{N+a}+\sqrt{N})^{2} \cdot c_{3} / c_{8}\right)}{\log \left((\sqrt{N+a}-\sqrt{N})^{2} \cdot c_{3} / c_{8}\right)}
\end{aligned}
$$

provided

$$
(\sqrt{N+a}-\sqrt{N})^{2} \cdot c_{3}<c_{8}<(\sqrt{N+a}+\sqrt{N})^{2} \cdot c_{3}
$$

It remains to show that $c_{3} / c_{8}=c_{10} / c_{9}$ or, equivalently, that

$$
\log \left(c_{8} \cdot c_{10}\right)=\log \left(c_{3} \cdot c_{9}\right) .
$$

Recalling the various definitions of our constants, the left-hand side of equation (7.2) becomes

$$
\begin{gathered}
\frac{2}{\phi(n)} \sum_{\substack{1 \leq r<n / 2 \\
(r, n)=1}}\left(\psi(1)-\psi\left(\frac{n-r}{n}\right)\right)+\frac{1}{\phi(n)} \sum_{\substack{1 \leq r<n / 2 \\
(r, n)=1}}\left(\psi\left(\frac{n-r}{n}\right)-\psi\left(\frac{r}{n}\right)\right) \\
=\frac{1}{\phi(n)} \sum_{\substack{1 \leq r<n / 2 \\
(r, n)=1}}\left(2 \psi(1)-\psi\left(\frac{r}{n}\right)-\psi\left(\frac{n-r}{n}\right)\right) .
\end{gathered}
$$


In order to express $\psi(z)$ with rational arguments as a finite combination of elementary functions, we appeal to a result of Gauss (see e.g. [4]):

$$
\begin{aligned}
\psi\left(\frac{r}{n}\right) & =-\gamma-\log n-\frac{1}{2} \pi \cot \left(\frac{\pi r}{n}\right) \\
& +\sum_{1 \leq k \leq n / 2}^{\prime} \cos (2 \pi k r / n) \log (2-2 \cos (2 \pi k / n)) .
\end{aligned}
$$

Here the prime over the summation indicates that only half the value associated with $k=n / 2$ is applied to the sum. The finite sum in (7.3) thus may be written as (again recalling that $\psi(1)=-\gamma$ )

$$
\log n-\frac{1}{\phi(n)} \sum_{\substack{1 \leq r<n \\(r, n)=1}} \sum_{1 \leq k \leq n / 2}^{\prime} \cos (2 \pi k r / n) \log (2-2 \cos (2 \pi k / n)) .
$$

Now, if we let $q(n)=\prod_{p \mid n} p$, then

$$
\sum_{\substack{1 \leq r<n / 2 \\(r, n)=1}} \cos \left(\frac{2 \pi k r}{n}\right)=\phi^{*}\left(\frac{(n, k)}{n} q(n)\right) \cdot \mu\left(\frac{n}{(n, k)}\right) \cdot \frac{n}{q(n)}
$$

where $\phi^{*}$ is the Euler totient for integer arguments, zero otherwise, and $\mu$ is the Möbius function. To see this, express the above sum in terms of primitive $n /(n, k)$ th roots of unity. Also, if $d>2$ is a fixed divisor of $n$,

$$
\begin{aligned}
\prod_{\substack{1 \leq k<n / 2 \\
(n, k)=n / d}}\left(2-2 \cos \left(\frac{2 k \pi}{n}\right)\right) & =\prod_{\substack{1 \leq j<d / 2 \\
(j, d)=1}}\left(2-2 \cos \left(\frac{2 j \pi}{d}\right)\right) \\
& =\prod_{\substack{1<j<d \\
(j, d)=1}}\left(1-e^{2 \pi i j / d}\right) .
\end{aligned}
$$

If $d$ is prime, then this is the product of the roots of the polynomial

$$
\frac{(x+1)^{d}-1}{x}=x^{d-1}+d x^{d-2}+\cdots+d
$$

and hence equal to $d$. If, however, $d$ has at least two distinct prime factors, then it is not difficult to show (see e.g. Washington [19, Prop. 2.8]) that

$$
\prod_{\substack{1 \leq j<d \\(j, d)=1}}\left(1-e^{2 \pi i j / d}\right)=1
$$

Collecting these facts together with (7.5) yields 


$$
\begin{aligned}
& \sum_{\substack{1 \leq r<n \\
(r, n)=1}} \sum_{\substack{1 \leq k<n / 2\\
}} \cos \left(\frac{2 \pi k r}{n}\right) \log (2-2 \cos (2 \pi k / n)) \\
= & \sum_{\substack{2<d \leq n \\
d \leq n}} \phi^{*}(q(n) / d) \cdot \mu(d) \cdot \frac{n}{q(n)} \cdot \log \left(\prod_{\substack{1 \leq k<n / 2 \\
(n, k)=n / d}}\left(2-2 \cos \left(\frac{2 k \pi}{n}\right)\right)\right) \\
= & -\sum_{\substack{p \mid n \\
p>2}} \phi(q(n) / p) \frac{n}{q(n)} \log (p) \\
= & -\sum_{\substack{p>n \\
p>2}} \frac{\phi(n)}{p-1} \cdot \log (p)
\end{aligned}
$$

where these last two sums are over $p$ prime.

This, with (7.3) and (7.4), implies that

$$
\log \left(c_{8} \cdot c_{10}\right)=\log n+\sum_{p \mid n} \frac{\log p}{p-1}=\log \left(n \mu_{n}\right)
$$

where $\mu_{n}=\prod_{p \mid n} p^{1 /(p-1)}$ (the contribution from $p=2$, for even $n$, is obtained by taking $k=n / 2$ in (7.4)). Now $c_{3} \cdot c_{9}$ satisfies

$$
\begin{aligned}
c_{3} \cdot c_{9} & =\prod_{p \mid n} p^{\max \left\{\operatorname{ord}_{p}(n / a)+\frac{1}{p-1}, 0\right\}+\min \left\{\operatorname{ord}_{p}(a), \operatorname{ord}_{p}(n)+\frac{1}{p-1}\right\}} \\
& =\prod_{p \mid n}^{p} p^{\operatorname{ord}_{p}(n)+\frac{1}{p-1}}=n \mu_{n}
\end{aligned}
$$

whence equality (7.2) holds, finishing the proof.

Application of this result with $n=3, s=1, a=3$ and $N=125$ enables one to obtain the bound (0.2) while other choices lead to a variety of examples, including those discussed in [6] and [12].

\section{Some APplications}

As the previous section indicates, it is possible to obtain versions of Theorem 1.1 (and Theorem 1.2, for that matter), for certain $m$, which are more explicit than that stated in $\$ 1$. Though we will not explore these aspects here, we note that Rickert's Theorem of [15] (compare to Theorem 1.1 with $n=m=2$ and $a_{u} \in\{-1,0,1\}$ ) essentially follows from the fact that the zeros $z_{0}, z_{1}$ and $z_{2}$ of polynomial (1.4) are well approximated by $-3 N / 2,-1 / \sqrt{3}$ and $1 / \sqrt{3}$, respectively.

In the case $n=m=2$, we obtain nontrivial bounds from Theorem 1.1 whenever $\lambda<2$. For larger values of $n$ or $m$, however, we require not only that $\lambda<n$, but also that $\lambda$ is smaller than that induced by any proper subset of the related numbers $\theta_{i}$ in $(0.1)(1 \leq i \leq m)$. By way of example, direct application of Theorem 1.1 with $n=3, m=2, N=8$, and $a_{i} \in\{-2,-1,0\}$ yields

$$
\max \left\{\left|\sqrt[3]{6}-\frac{p_{1}}{q}\right|,\left|\sqrt[3]{7}-\frac{p_{2}}{q}\right|\right\}>q^{-2.66985}
$$


Table 1: Cases of Theorem 1.1 for $m=2$ :

\begin{tabular}{|c|c|c|c|c|c|}
\hline$\theta_{1}, \theta_{2}$ & $N, a_{i} \neq 0$ & $\lambda$ & $\theta_{1}, \theta_{2}$ & $N, a_{i} \neq 0$ & $\lambda$ \\
\hline$\sqrt{2}, \sqrt{3}$ & $2,-1,1$ & 1.79155 & $\sqrt{10}, \sqrt{11}$ & $3^{2}, 1,2$ & 1.98873 \\
\hline$\sqrt{2}, \sqrt{7}$ & $82^{2}, 3,4$ & 1.88908 & $\sqrt{10}, \sqrt{22}$ & $136^{2},-6,6$ & 1.76160 \\
\hline$\sqrt{2}, \sqrt{11}$ & $10^{2},-2,-1$ & 1.74788 & $\sqrt{10}, \sqrt{31}$ & $22^{2}, 6,12$ & 1.99858 \\
\hline & $4^{2}, 1,2$ & 1.89813 & $\sqrt{10}, \sqrt{38}$ & $6^{2}, 2,4$ & 1.88331 \\
\hline$\sqrt{2}, \sqrt{23}$ & $24^{2},-1,2$ & 1.97500 & $\sqrt{14}, \sqrt{15}$ & $4^{2},-2,-1$ & 1.91627 \\
\hline$\sqrt{2}, \sqrt{29}$ & $140^{2}, 2,4$ & 1.62588 & $\sqrt{15}, \sqrt{17}$ & $4^{2},-1,1$ & 1.90671 \\
\hline$\sqrt{2}, \sqrt{51}$ & $7^{2}, 1,2$ & 1.79008 & $\sqrt{19}, \sqrt{22}$ & $61^{2},-3,3$ & 1.79694 \\
\hline$\sqrt{3}, \sqrt{5}$ & $123^{2},-6,-4$ & 1.82227 & $\sqrt{21}, \sqrt{23}$ & $5^{2},-4,-2$ & 1.96101 \\
\hline$\sqrt{3}, \sqrt{23}$ & $5^{2},-2,2$ & 1.94595 & $\sqrt{23}, \sqrt{47}$ & $48^{2},-4,-1$ & 1.95362 \\
\hline & $5^{2}, 1,2$ & 1.8 & $\sqrt{34}, \sqrt{35}$ & $6^{2},-2,-1$ & 1.81853 \\
\hline & $5^{2}, 2$ & 1 & $\sqrt{34}, \sqrt{ }$ & $6^{2},-2,2$ & 037 \\
\hline & $71^{2},-1,2$ & 1.83947 & $\sqrt{35}, \sqrt{37}$ & & 1.81607 \\
\hline$\sqrt{3}, \sqrt{47}$ & $7^{2},-2,-1$ & 1.79307 & $\sqrt{37}, \sqrt{38}$ & $6^{2}, 1,2$ & 1.81372 \\
\hline$\sqrt{5}, \sqrt{11}$ & $199^{2},-1,4$ & 1.90617 & $\sqrt[3]{3}, \sqrt[3]{21}$ & $3^{3},-6,-3$ & 2.57831 \\
\hline$\sqrt{5}, \sqrt{47}$ & $7^{2},-4,-2$ & 1.85791 & $\sqrt[3]{3}, \sqrt[3]{30}$ & $3^{3},-3,3$ & 2.50876 \\
\hline$\sqrt{5}, \sqrt{79}$ & $9^{2},-2,-1$ & 1.75964 & $\sqrt[3]{5}, \sqrt[3]{9}$ & $3^{3},-3,-2$ & 2.52360 \\
\hline & $9^{2},-1,1$ & 1.75893 & $\sqrt[3]{7}, \sqrt[3]{9}$ & $2^{3},-1,1$ & 2.57831 \\
\hline$\sqrt{5}$ & $29^{2},-4,4$ & 1.82863 & $\sqrt[3]{22}, \sqrt[3]{43}$ & $14^{3}, 6,8$ & 2.22145 \\
\hline$\sqrt{6}$ & $5^{2},-2,-1$ & 1.85558 & $\sqrt[3]{23}, \sqrt[3]{25}$ & $3^{3},-4,-2$ & 2.78961 \\
\hline & $5^{2},-1,1$ & 1.85115 & $\sqrt[3]{25}, \sqrt[3]{26}$ & $3^{3},-2,-1$ & 2.13916 \\
\hline & $169^{2},-5,5$ & 1.79760 & $\sqrt[3]{25}, \sqrt[3]{29}$ & $3^{3},-2,2$ & 2.73786 \\
\hline & $37^{2},-1,3$ & 1.99312 & $\sqrt[3]{26}, \sqrt[3]{28}$ & $3^{3},-1,1$ & 2.13097 \\
\hline & $37^{2}, 3,6$ & 1.84857 & $\sqrt[3]{28}, \sqrt[3]{29}$ & $3^{3}, 1,2$ & 2.12328 \\
\hline & $8^{2},-2,-1$ & 1.77425 & $\sqrt[3]{29}, \sqrt[3]{30}$ & $3^{3}$ & 2.48614 \\
\hline$\sqrt{7}, \sqrt{65}$ & $8^{2},-1,1$ & 1.77324 & $\sqrt[3]{29}, \sqrt[3]{31}$ & $3^{3}, 2,4$ & 2.69341 \\
\hline$\sqrt{7}, \sqrt{83}$ & $82^{2},-1,3$ & 1.88907 & $\sqrt[3]{30}, \sqrt[3]{33}$ & $3^{3}, 3,6$ & 2.45379 \\
\hline
\end{tabular}

for $q \geq q_{0}$ effective. If we instead consider $n=3, m=1, N=101847558$ and $a_{1}=5$, we find (see Chudnovsky $[6$, Table 1$]$ )

$$
\left|\sqrt[3]{6}-\frac{p}{q}\right|>q^{-2.32056}
$$

for $q \geq q_{1}$ effectively computable, so that (8.1) is in fact weaker than the latter result.

In Table 1, we collect examples of bounds of the form (0.1) for pairs of square roots and cube roots of fairly small integers. We find these examples by considering integers "close" to squares (or cubes) which themselves possess "large" square (or cubic) factors. For instance, we obtain a simultaneous measure for $(\sqrt{3}, \sqrt{5})$ because $123^{2}=3 \cdot 71^{2}+6=5 \cdot 55^{2}+4$. It is not difficult to show that if Theorem 1.1 provides a bound for $\left(\theta_{1}, \theta_{2}\right)=(\sqrt{A}, \sqrt{B})$, then it yields like bounds for $(\sqrt{A}, \sqrt{A B})$ and $(\sqrt{B}, \sqrt{A B})$ with the same value for $\lambda$. For example, we have, taking $N=49$, and $a_{i} \in\{-1,0,1\}$, 


$$
\max \left\{\left|\sqrt{2}-\frac{p_{1}}{q}\right|,\left|\sqrt{3}-\frac{p_{2}}{q}\right|\right\}>q^{-1.79155}\left(q \geq q_{0}\right)
$$

while $N=100$ and $a_{i} \in\{-4,-2,0\}$ gives

$$
\max \left\{\left|\sqrt{2}-\frac{p_{1}}{q}\right|,\left|\sqrt{6}-\frac{p_{2}}{q}\right|\right\}>q^{-1.79155}\left(q \geq q_{1}\right)
$$

and $N=144$ and $a_{i} \in\{0,3,6\}$ yields

$$
\max \left\{\left|\sqrt{3}-\frac{p_{1}}{q}\right|,\left|\sqrt{6}-\frac{p_{2}}{q}\right|\right\}>q^{-1.79155}\left(q \geq q_{2}\right) .
$$

We will restrict ourselves in Table 1 to listing only examples which are "primitive" in this sense (to avoid duplication). Analogous results to this hold for other values of $n$ and $m$ and it is of interest to note that while the measure produced from Theorem 1.1 (taking $N=8$ and $\left.a_{i} \in\{0,1,2\}\right)$ for $\left(\theta_{1}, \theta_{2}\right)=(\sqrt[3]{9}, \sqrt[3]{10})$ is weaker than that obtained for $\sqrt[3]{10}$ alone, the same is not known to be the case for the related pair $(\sqrt[3]{3}, \sqrt[3]{30})$.

For larger values of $m$, a couple of interesting examples are

$$
\max \left\{\left|\sqrt{2}-\frac{p_{1}}{q}\right|,\left|\sqrt{3}-\frac{p_{2}}{q}\right|,\left|\sqrt{47}-\frac{p_{3}}{q}\right|,\left|\sqrt{51}-\frac{p_{4}}{q}\right|\right\}>q^{-1.67429}
$$

for $q \geq q_{0}$, and

$$
\max \left\{\left|\sqrt[3]{25}-\frac{p_{1}}{q}\right|,\left|\sqrt[3]{26}-\frac{p_{2}}{q}\right|,\left|\sqrt[3]{28}-\frac{p_{3}}{q}\right|,\left|\sqrt[3]{29}-\frac{p_{4}}{q}\right|\right\}>q^{-1.86545}
$$

for $q \geq q_{1}$, where both $q_{0}$ and $q_{1}$ are effective. These follow by taking $N=49, a_{u} \in$ $\{-2,-1,0,1,2\}, n=2$ and $N=27, a_{u} \in\{-2,-1,0,1,2\}, n=3$, respectively, in Theorem 1.1 .

It appears to be rather harder to find "attractive" examples of the utility of Theorem 1.2. Part of the difficulty is that if $\theta_{1}, \ldots, \theta_{m}$ are elements of a real field of degree $m+1$ over $\mathbb{Q}$ with $1, \theta_{1}, \ldots, \theta_{m}$ linearly independent over $\mathbb{Q}$, then

$$
\left|x_{0}+x_{1} \theta_{1}+\cdots+x_{m} \theta_{m}\right|>X^{-m}
$$

for sufficiently large $X=\max _{0 \leq j \leq m}\left|x_{i}\right|$, via consideration of norms (see e.g. Cassels [5, Chapter V]). We are therefore only interested in bounds upon linear forms which sharpen this. One such result follows from Theorem 1.2 with $n=m=2, N=816^{2}$ and $a_{i} \in\{0,2,4\}$, whence

$$
|x+y \sqrt{2}+z \sqrt{985}|>X^{-2.89514}
$$

for $x, y$ and $z$ integers, $X=\max \{|x|,|y|,|z|\}$ and $X \geq X_{0}$ effectively computable. We may also attain this bound via transference from Theorem 1.1 (from which one obtains $\lambda=1.59144$ as a simultaneous irrationality measure for $(\sqrt{2}, \sqrt{985})$ ), with direct application of Theorem 1.2 providing an improvement only noticeable in the sixth decimal place of $\lambda_{1}$. In general, Theorem 1.2 represents only a slight sharpening of the corresponding transference result if the $a_{i}$ 's are "evenly" distributed, but is more effective otherwise.

The problem of solving simultaneous Pell's equations is discussed in [14], motivated by the desire to find elliptic curves over $\mathbb{Q}(\sqrt{5})$ with good reduction away from 
the prime 2, and in [18], related to solutions of Thue equations. Results obtained from Theorem 1.1 may be applied to this problem. For instance, to solve

$$
x^{2}-3 z^{2}=u, y^{2}-5 z^{2}=v
$$

we use the bound

$$
\max \left\{\left|\sqrt{3}-\frac{p_{1}}{q}\right|,\left|\sqrt{5}-\frac{p_{2}}{q}\right|\right\}>q^{-1.82227}
$$

$\left(q \geq q_{0}\right)$ from Table 1. Arguing as in [15], one may deduce that

$$
\max \{|x|,|y|,|z|\} \leq k_{0}(\max \{|u|,|v|\})^{5.62652}
$$

for an effectively computable absolute constant $k_{0}$. For fixed $u$ and $v$, this enables us to find all solutions to (8.2). The examples considered by Rickert [15] are the pair of simultaneous equations

$$
x^{2}-2 z^{2}=u, y^{2}-3 z^{2}=v
$$

and

$$
x^{2}-2 z^{2}=u, y^{2}-58 z^{2}=v .
$$

For these, we obtain

$$
\max \{|x|,|y|,|z|\} \leq k_{1}(\max \{|u|,|v|\})^{4.79732}
$$

and

$$
\max \{|x|,|y|,|z|\} \leq k_{2}(\max \{|u|,|v|\})^{2.67294}
$$

respectively, where $k_{1}$ and $k_{2}$ are effective. Similar results for simultaneous Pelltype equations (of greater degree) may also be readily produced.

\section{NORM FORM EQUATIONS}

In [10], Fel'dman considers norm form equations

$$
N_{K / \mathbb{Q}}\left(x_{0} w_{0}+\cdots+x_{m} w_{m}\right)=f\left(x_{0}, \ldots, x_{m}\right)
$$

where $w_{0}, \ldots, w_{m}$ are certain algebraic numbers, $f$ a polynomial of small degree and $K=\mathbb{Q}\left(w_{0}, \ldots, w_{m}\right)$. To solve these, he essentially utilizes a version of Theorem 1.2 to simultaneously approximate the $w_{i}$ 's. We will show that a slight refinement of his techniques, utilizing both Theorems 1.1 and 1.2, enables one to effectively solve the equation ( $u$ constant)

$$
N_{K / \mathbb{Q}}\left(x+y \sqrt[4]{M^{4}-1}+z \sqrt[4]{M^{4}+1}\right)=u
$$

for all $M \geq 6$. Similarly, we find all solutions to

$$
N_{K / \mathbb{Q}}\left(x+y \sqrt[6]{M^{6}-1}+z \sqrt[6]{M^{6}+1}\right)=u
$$

for $M \geq 4$.

Let us concentrate on equation (9.1); the argument is essentially unchanged for (9.2). We will require the observation that Theorems 1.1 and 1.2 remain valid if $p_{0}, \ldots, p_{m}, q$ and $x_{0}, \ldots, x_{m}$ are taken to be integers in a fixed imaginary quadratic field (see Fel'dman [9]). Let

$$
L=x+y \sqrt[4]{M^{4}-1}+z \sqrt[4]{M^{4}+1}
$$


for $x, y$ and $z$ integers, not all zero, and let $L_{s, t}(0 \leq s, t \leq 3)$ be the conjugates of $L$ associated with the extension $K=\mathbb{Q}\left(\sqrt[4]{M^{4}-1}, \sqrt[4]{M^{4}+1}\right)$, as given by

$$
L_{s, t}=x+y \sqrt[4]{M^{4}-1} i^{s}+z \sqrt[4]{M^{4}+1} i^{t} .
$$

Reasoning as in $[10, \S 3]$, all but four of these conjugates satisfy

$$
\left|L_{s, t}\right| \geq c_{0} X
$$

for $c_{0}$ effective, $X=\max \{|x|,|y|,|z|\}$. Let $L_{i}(1 \leq i \leq 4)$ be the remaining conjugates, ordered so that

$$
\left|L_{1}\right| \leq\left|L_{2}\right| \leq\left|L_{3}\right| \leq\left|L_{4}\right| \text {. }
$$

We apply Theorem 1.2 (with $x, y, z \in \mathbb{Z}[i]$ ) to estimate the smallest conjugate, finding that

$$
\left|L_{1}\right|>X^{-6.35740}
$$

for $X \geq X_{0}$ and $M \geq 6$. It follows from (9.3) that

$$
\left|L_{2}\right| \geq \frac{1}{2}\left|L_{2}-L_{1}\right|=\frac{1}{2}\left|y \sqrt[4]{M^{4}-1}\left(i^{s_{1}}-i^{s_{2}}\right)+z \sqrt[4]{M^{4}+1}\left(i^{t_{1}}-i^{t_{2}}\right)\right| .
$$

Applying Theorem 1.1 (more specifically, Theorem 7.1 with $p, q \in \mathbb{Z}[i]$ ), we have

$$
\left|\sqrt[4]{1+\frac{2}{M^{4}-1}}-\frac{p}{q}\right|>|q|^{-2.49951}
$$

for $|q| \geq q_{0}$ and $M \geq 6$. Thus (9.7) implies

$$
\left|L_{2}\right|>X^{-1.49952}
$$

for $X \geq X_{1}$ and $M \geq 6$. Inequalities (9.4), (9.5), (9.6) and (9.8) combine to yield

$$
\left|N_{K / \mathbb{Q}}\left(x+y \sqrt[4]{M^{4}-1}+z \sqrt[4]{M^{4}+1}\right)\right|=\left|\prod_{0 \leq s, t \leq 3} L_{s, t}\right|>X^{1.14405}
$$

for $X \geq X_{2}$ and $M \geq 6$, which enables one to solve (9.1) as desired.

If we replace the constant $u$ in equation (9.1) or (9.2) by a polynomial in $\mathbb{Z}[x, y, z]$ of fixed degree, then the preceding argument may permit solution of the resulting equation, provided $M$ is large enough. For example, to solve

$$
N_{K / \mathbb{Q}}\left(x+y \sqrt[4]{M^{4}-1}+z \sqrt[4]{M^{4}+1}\right)=f(x, y, z)
$$

we require only that $M$ be bounded as follows.

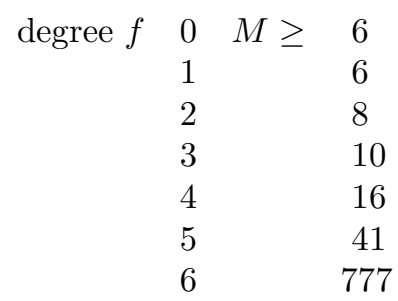

In essence, in this situation, we are dealing with a Diophantine inequality of the form

which we may solve provided $\delta<7$.

$$
\left|N_{K / \mathbb{Q}}\left(x+y \sqrt[4]{M^{4}-1}+z \sqrt[4]{M^{4}+1}\right)\right|<X^{\delta}
$$




\section{ACKNOWLEDGEMENTS}

The author would like to thank Professor Cameron Stewart for many helpful discussions on the contents of this paper. Thanks are also due to Carolyn Jackson for her precise typing and to Ryan English for preparation of Figure 1.

$$
\max \left\{\left|\theta_{1}-\frac{p_{1}}{q}\right|,\left|\theta_{2}-\frac{p_{2}}{q}\right|\right\}>q^{-\lambda}
$$

for $q \geq q_{0}\left(\theta_{1}, \theta_{2}, \lambda\right)$.

Note added in proof. It has come to the author's attention that an analogous result to our Theorem 1.2 has appeared in a paper by A. Dubitskas (Estimation of a linear form in algebraic numbers, Lithuanian Math. J. 31 (1991), 56-61).

\section{REFERENCES}

1. A. Baker, Rational approximations to certain algebraic numbers, Proc. London Math. Soc. (3) 14 (1964), 385-398. MR 28:5029

2. _ Rational approximations to $\sqrt[3]{2}$ and other algebraic numbers. Quart. J. Math. Oxford Ser. (2) 15 (1964), 375-383. MR 30:1977

3. Simultaneous rational approximations to certain algebraic numbers, Proc. Cambridge Philos. Soc. 63 (1967), 693-702. MR 35:4167

4. H. Bateman and A. Erdélyi, Higher Transcendental Functions, McGraw-Hill, 1953, v.1.

5. J.W.S. Cassels, An Introduction to Diophantine Approximation, Cambridge University Press, Cambridge, 1957. MR 19:396h

6. G.V. Chudnovsky, On the method of Thue-Siegel, Ann. of Math., (2) 117 (1983), 325-382. MR 85g: 11058

7. J. Dieudonné, Calcul infinitésimal, Hermann, Paris, 1968. MR 37:2557

8. D. Easton, Effective irrationality measures for certain algebraic numbers, Math. Comp. 46, no. 174 (1986), 613-622. MR 87f:11047

9. N.I. Fel'dman, An estimate of an incomplete linear form in several algebraic variables, Math. Notes 7 (1970), 343-349. MR 42:3026

10. Effective bounds for the number of solutions of certain diophantine equations. Math. Notes 8 (1970), 674-679. MR 42:7590

11. M. Hata, Rational approximation to $\pi$ and some other numbers, Acta Arith. 63 (1993), 335349. MR 94e: 11082

12. A. Heimonen, T. Matala-aho and K. Väänänen, An application of Jacobi type polynomials to irrationality measures, Bull. Austral. Math. Soc. 50 (1994), 225-243. MR 95:02

13. C.F. Osgood, The simultaneous diophantine approximation of certain kth roots, Proc. Cambridge Philos. Soc. 67 (1970), 75-86. MR 43:1927

14. R.G.E. Pinch, Simultaneous Pellian equations, Proc. Cambridge Philos. Soc. 103 (1988), 35-46. MR 89a:11029

15. J.H. Rickert, Simultaneous rational approximations and related diophantine equations, Proc. Cambridge Philos. Soc. 113 (1993), 461-472. MR 94i:11043

16. K.R. Roth, Rational approximations to algebraic numbers, Mathematika 2 (1955), 1-20: corrigendum, ibid. 168. MR 17:1060d; MR 17:242d

17. W.M. Schmidt, Simultaneous approximation to algebraic numbers by rationals, Acta Math. 125 (1970), 189-201. MR 42:3028

18. N. Tzanakis, Explicit solution of a class of quartic Thue equations, Acta Arith. 64 (1993), 271-283. MR 94e:11022

19. L. Washington, Introduction to cyclotomic fields, Springer-Verlag, GTM 83, 1982. MR 85g:11001

Department of Pure Mathematics, The University of Waterloo, Waterloo, Ontario, CANADA, N2L 3G1

Current address: Department of Mathematics, University of Michigan, Ann Arbor, Michigan 48109

E-mail address: mabennet@math.1sa.umich.edu 